\title{
Renal Cell Carcinoma Embolization for the Treatment of Budd-Chiari Syndrome
}

\author{
Stephen Allison ${ }^{1}$ Guy Johnson ${ }^{1}$ Matthew Kogut \\ ${ }^{1}$ University of Washington, Seattle, Washington, United States \\ J Clin Interv Radiol ISVIR 2017;1:134-136.
}

\author{
Eric Monroe ${ }^{1}$
}

Address for correspondence Stephen Allison, MD, University of Washington, 1959 NE Pacific Street, Seattle, WA 98195, United States (e-mail: sallison@uw.edu).

\begin{abstract}
Renal cell carcinoma (RCC) is the eighth most common malignancy, accounting for $3 \%$ of all newly diagnosed neoplasms. RCC venous invasion occurs in 4 to $10 \%$ of cases. We

Keywords

- renal cell carcinoma

- embolization

- Budd-Chiari syndrome

- Stauffer's syndrome report a case of RCC with extensive vascular invasion and new-onset liver dysfunction. The etiology of the liver dysfunction was attributed to hepatic vein obstruction; herein we describe the first reported case of transcatheter embolization of RCC for BuddChiari syndrome.
\end{abstract}

\section{Introduction}

Renal cell carcinoma (RCC) is the most common renal malignancy comprising $85 \%$ of all malignant renal tumors. Venous invasion occurs in 4 to $10 \%$ of patients with renal neoplasms, and within this group, 2 to $16 \%$ have tumors extending into the right atrium. ${ }^{1}$ The presence of venous extension connotes stage III disease in the Robson classification and T3 tumor in the TNM staging system. T3 tumors are further subdivided into the degree of central extension of the tumor thrombus with important implications for surgical management. Tumor with venous invasion extending to the supradiaphragmatic inferior vena cava (IVC) is T3c.

When possible, surgery with complete resection of tumor and the associated thrombi may be attempted with curative intent. Unfortunately, many patients are not surgical candidates at the time of diagnosis. Renal artery embolization has been proposed as a palliative treatment for unresectable RCC in patients unfit or unwilling to undergo surgery. ${ }^{2}$ Renal artery embolization for RCC was first described in 1969; since then, various techniques and embolic materials have been described.

We present a case in which we performed an embolization for a RCC with T3c tumor thrombus. The patient had severe coagulopathy (international normalized ratio [INR] 2.8) secondary to rapidly progressive liver dysfunction and was declined for surgical intervention. The goal was to devascularize and shrink tumor thrombus, thereby improv- ing hepatic venous outflow. This technical review was performed according to the World Medical Association Declaration of Helsinki.

\section{Technique}

A 70-year-old female patient presented with acute-onset weakness, dyspnea, and decrease in appetite with an associated $10 \mathrm{lb}$ weight loss. She also reported hematochezia. A colonoscopy was unrevealing. Initial laboratory evaluation revealed her liver function tests were aspartate aminotransferase (AST) 1,161 and alanine aminotransferase (ALT) 773. Abdominal ultrasound demonstrated a large right renal mass with extension into the IVC. Subsequent computed tomography (CT) scan demonstrated a $6 \mathrm{~cm}$ right lower pole renal mass with tumor extension into the IVC and right atrium (-Fig. 1a, b). An echocardiogram confirmed a 2 to $3 \mathrm{~cm}$ right atrial mass consistent with tumor thrombus.

The patient was evaluated by the tumor board, including the urologist, oncologist, and nephrologist, and given the high risk of mortality in such a complex surgery, the patient was referred to interventional radiology for tumor embolization, in the hope that would result in tumor shrinkage and recanalization of the thrombus.

The patient consented for the procedure after being explained the risks and benefits. After gaining access via the right common femoral artery, the right renal artery was selected and subsequently, the lower pole segmental renal 


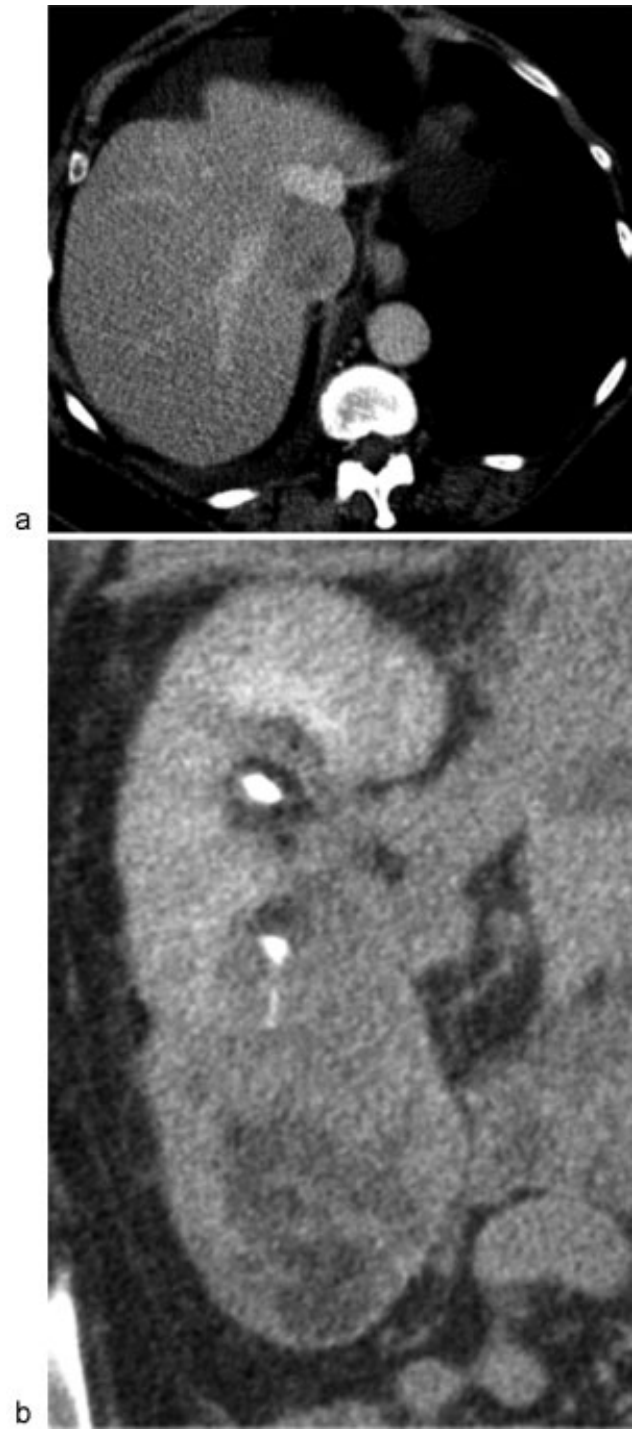

Fig. 1 (a, b) CT images demonstrating tumor thrombus within the intrahepatic IVC and right renal lower pole mass. CT, computed tomography; IVC, inferior vena cava.

artery. Obvious tumor vascularity in the lower pole was noted, extending into the right renal vein and IVC (-Fig. 2a). Embolization was performed using 150 to 250 polyvinyl alcohol particles to stasis (-Fig. 2b).

After embolization, there was a notable improvement in her laboratories with an AST 41 and ALT 63 after 14 days (-Fig. 3). There was also gradual improvement of her INR during her hospitalization, which decreased to 1.5 (from 2.8) in the same time frame. Repeat CT scan only 5 days after embolization demonstrated axial intrahepatic IVC crosssectional diameter decreased from 44 to $39 \mathrm{~mm}$.

Although the acute phase of Budd-Chiari syndrome (BCS) resolved, the patient was deemed to be extremely deconditioned due to her complex medical problems, renal tumor, and thrombus burden. The patient was ultimately discharged to a skilled nursing facility with plans for palliative care.

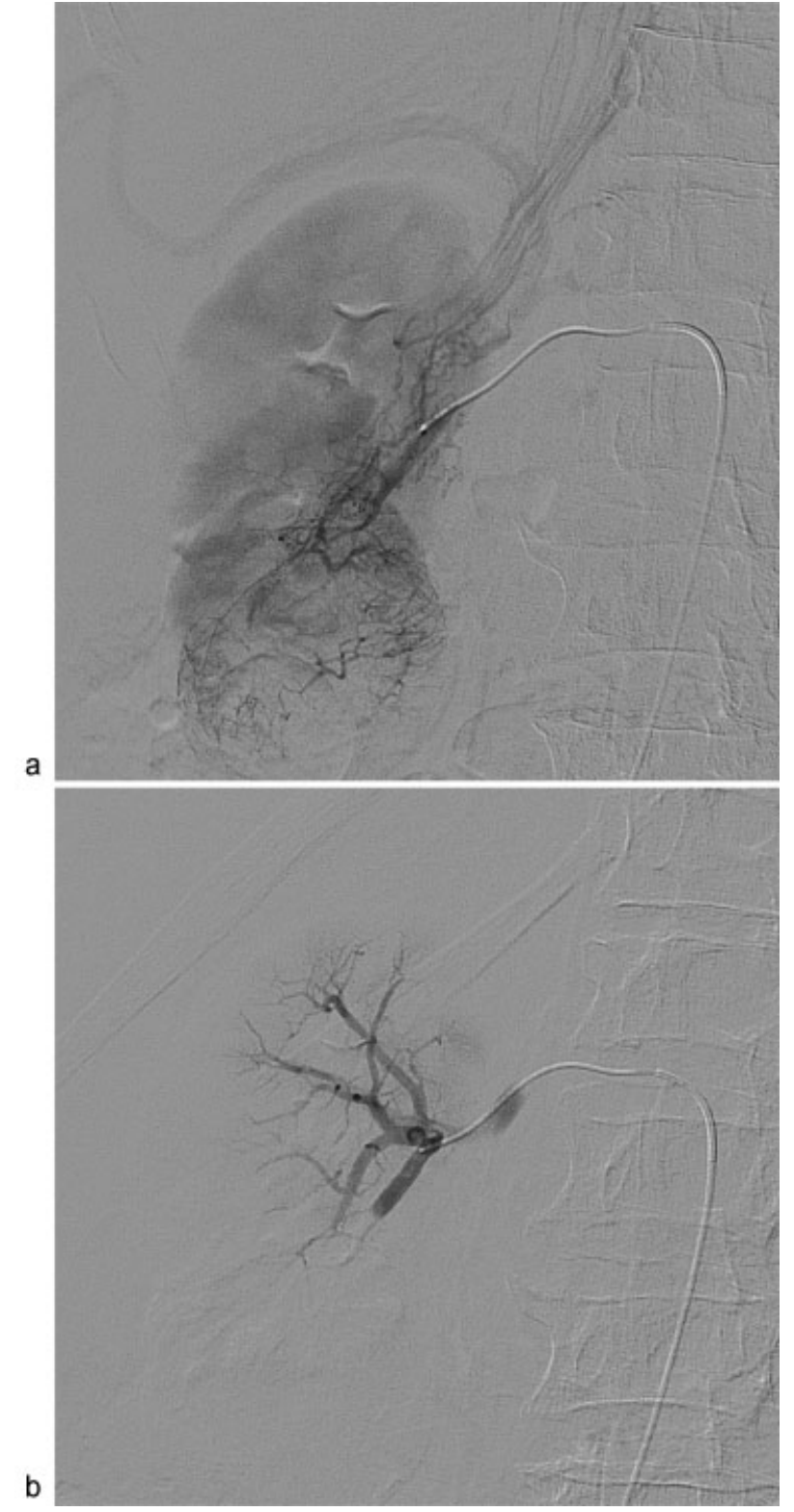

Fig. 2 (a, b) Digital subtracted angiography image demonstrating tumor vascularity within the right renal lower pole and tumor thrombus extending into the IVC (a) and postembolization DSA image demonstrating no residual tumor (b). IVC, inferior vena cava; DSA, digital subtracted angiography.

\section{Discussion}

BCS is defined as obstruction of the hepatic veins resulting in congestive hepatopathy. The clinical presentation can vary from asymptomatic radiographic findings to severe fulminant hepatic failure. The classic triad of presentation is abdominal pain, ascites, and hepatomegaly. ${ }^{3}$ The etiology can be divided into primary and secondary causes. Primary BCS results from the direct occlusion of hepatic veins from an intraluminal source of the thrombus (most common). Secondary BCS is caused by an extrinsic compression of either the IVC or the hepatic veins. ${ }^{3}$

RCC, along with hepatocellular carcinoma and adrenal tumors, are the major malignant conditions capable of direct 


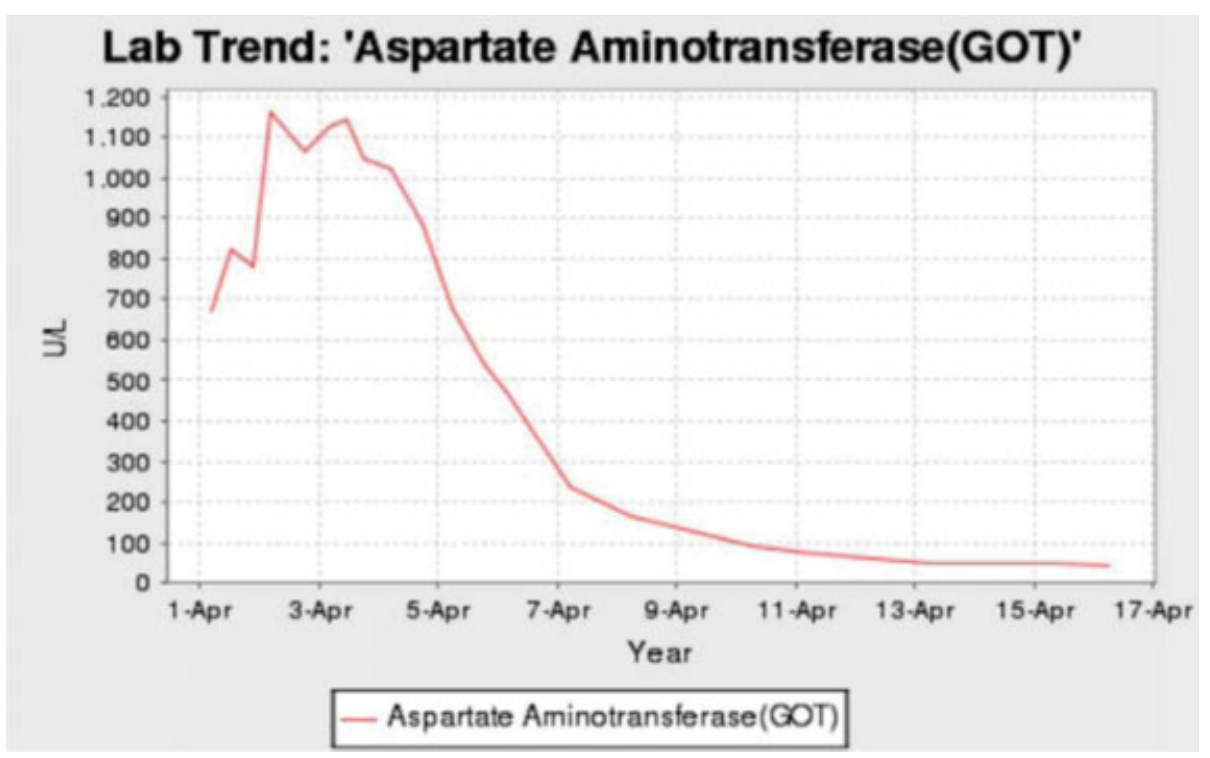

Fig. 3 Graph of liver enzyme AST/GOT before and after embolization. AST/GOT, aspartate aminotransferase.

IVC invasion and venous obstruction. Once the IVC is obliterated, collateral veins may develop to reestablish venous return to the right atrium. Collaterals drain via the azygous vein using its extensive communications with the lumbar and renal veins, or extensive perirenal collaterals. ${ }^{4}$

Usually, the ideal treatment for advanced RCC causing BCS is surgical excision with removal of the tumor thrombus from the IVC and/or hepatic veins in a select group of patients. In other patients, treatment for BCS may include anticoagulation and percutaneous angioplasty with stenting. This treatment may be harmful, with the potential of dislodging a fragment of the tumor resulting in pulmonary embolism. ${ }^{3}$ Surgery before developing decompensated liver disease is paramount. Once the patient develops hepatic failure following hepatic vein involvement, the outcome is poor. Kume et $\mathrm{al}^{5}$ reported four cases presenting at their center over 7 years along with eight cases reported in international literature. Seven patients had liver failure at the time of diagnosis, and surgical resection was possible in only one patient.

BCS caused by RCC can be classified into two groups: with or without acute liver failure. There are no reports in the literature that describe survival in those patients presenting with acute liver failure secondary to BCS from RCC. Severe sudden onset of BCS is not a common presentation of RCC and has a very poor prognosis. ${ }^{6}$

However, our patient's liver function improved dramatically after embolization. Repeat CT scan demonstrated a slight decrease in the size of the intracaval tumor thrombus. We hypothesize that this slight size change allowed enough room for the hepatic veins to decompress and resolve the BCS. However, an alternative explanation for the patient's presentation was considered. In 1961, Dr. Maurice Stauffer, an American gastroenterologist, recorded liver dysfunction in a patient with RCC without hepatic metastases. ${ }^{7}$ Stauffer's syndrome, seen in 3 to $20 \%$ of RCC patients, is characterized by elevations in liver enzymes as well as abnormal levels of hepatic synthetic products. Elevations of liver enzymes and prothrombin time exist in $66 \%$ of cases but usually resolve with nephrectomy. ${ }^{7}$

RCC embolization was an effective means to bridge the gap between liver decomposition and the time of surgery. To our knowledge, this is the first reported case of RCC transcatheter embolization for liver failure secondary to BCS.

\section{Conflict of Interest}

On behalf of all authors, the corresponding author states that there is no conflict of interest.

\section{References}

1 Nouh MA, Inui M, Kakehi Y. Renal cell carcinoma with IVC thrombi; current concepts and future perspectives. Clin Med Oncol 2008;2:247-256

2 Maxwell NJ, Saleem Amer N, Rogers E, Kiely D, Sweeney P, Brady AP. Renal artery embolisation in the palliative treatment of renal carcinoma. Br J Radiol 2007;80(950):96-102

3 Shirodkar SP, Soloway MS, Ciancio G. Budd-Chiari syndrome in urology: Impact on nephrectomy for advanced renal cell carcinoma. Indian J Urol 2011;27(03):351-356

4 Sarwar S, Khan AA. Budd Chiari syndrome: a rare complication of renal cancer. J Coll Physicians Surg Pak 2008;18(11):726-727

5 Kume H, Kameyama S, Kasuya Y, Tajima A, Kawabe K. Surgical treatment of renal cell carcinoma associated with Budd-Chiari syndrome: report of four cases and review of the literature. Eur J Surg Oncol 1999;25(01):71-75

6 Shih KL, Yen HH, Su WW, Soon MS, Hsia CH, Lin YM. Fulminant Budd-Chiari syndrome caused by renal cell carcinoma with hepatic vein invasion: report of a case. Eur J Gastroenterol Hepatol 2009;21(02):222-224

7 Palapattu GS, Kristo B, Rajfer J. Paraneoplastic syndromes in urologic malignancy: the many faces of renal cell carcinoma. Rev Urol 2002;4(04):163-170 